UDC $1 / 14$

Haig KHATCHADOURIAN

\title{
TIME AND CHANGE*
}

I

Elsewhere ${ }^{1}$ I attempted a very partial analysis of the ordinary concept of time, and the logical relation between time and change, and motion. Here I shall attempt a more extended analysis of (1) that concept - arrived at by abstraction from the ordinary uses of 'time,' including the uses of 'the past', 'the present ${ }_{2}$ ', 'a minute 2 ', 'an hour', etc.; (2) concept of the passage of time, $(P S)$, including the concepts past, present, future, etc., and those of seconds, minutes, hours, days, etc. And (3) the much more refined and enriched and in some ways modified concept of "real time" ultimately based on the ordinary concept of time.

The concepts past, present, future are logical components of the overall concept of time1, and demarcate parts of time-1's passage, so long as matter and/or energy exist; for example, in some actual universe, even if such a universe lacked all intelligence, such as human life; hence if no living thing knew about, or experienced, the distinction - and the difference between - "past", "present" and "future".

The general concept of time - in contrast to its sub-concept of time's passage - is not a metric concept. Otherwise it would have made sense to ask: "How many centuries, millennia, or aeons-long is time?"”2. But physicists and cosmo-

This article was written by late Professor Haig Khatchadourian in October 2, 2015.

1 See (Khatchadourian, 1961).

2 But if (real)time had a beginning - if it began with the very beginning of the universe, e.g., with the quantum logists speak perfectly sensibly of (a) time in relation to the existence of the universe, when they maintain that time came into existence when the universe came into existence; that is, that time began or came into existence (at least) during the quantum fluctuations in a vacuum, at (possibly) ${ }^{-43}$ Planck time, hence before the Big Bang itself; hence that at least 16.5 billion years have passed since time began. But note that the idea of the length of time does not apply to the concepts of the past, the present, and the future themselves. In other words, time is not a selfreflexive concept; if we treat it as self-reflexive, we would have a vicious infinite regress on our hands.

Time is presupposed in any change of any kind since the latter presupposes the passage of time, and the latter presupposes time. More simply stated, " $X$ is changing" presupposes " $X$ is in time 1 ", The same is true of an event or happening. But note that we do not speak of an event or happening itself as changing; nor do we say that change occurs (or can occur) in an event or occurrence. But I think that an event may be a change in the ordinary meaning of 'change'. Some occurrences or happenings other than events may also change in something or other, or a change of something into something else. As stated earlier, past, present and future are objective realities in the universe, independent of us; a causal result of the quantum fluctuations that,

fluctuations that is commonly believed by scientists to have given rise to the Big Bang, then that question would make sense.

3 Contrast 'the past', 'the present', 'the future'. 
according to recent speculations, that caused the Big Bang while the measurements of the passage of time are a human invention. This leads to the question whether the passage of time, hence time itself, would cease to be if at some future point all changes or physical processes in the universe were to stop or cease, to come to an end. I think the answer is No: that time would continue to pass; though I believe that any existing sentient beings in the universe would then lose all sense of time, and so, of the passage of time.

In contrast to the foregoing, philosophers generally use 'change' in a broad sense as a generic label for all events, occurrences, etc., as well as to things we ordinarily call changes. For convenience I shall follow general philosophical usage in this paper, except where ordinary usage is more appropriate.

An entity's existence in time is not any sort of change in it or in any other entity. A change occurs at some rate or other; but the concept of the rate of change is inapplicable to anything enduring in time. Objects, events and occurrences change in the ordinary meaning of 'change', in quality, amount, intensity, etc., at some temporal rate or other. Similarly, with events, occurrences, etc. Consequently, the existence of anything in time is also an event, occurrence or happening in the ordinary meaning of these words.

Note that, at any given moment it makes no sense to ask: "How long is the past?" but that it makes sense to ask how much longer time now is than it was five, ten or twenty hours, etc., since the passing of those hours, etc. For example, it makes no sense to say: "The past is now, on August 1, 2015, two years longer than it was on August 1, 2013"4. Consequently the fact that we

4 This should not be confused with e.g. the fact that we meaningfully speak of some particular occurrence or period, such as, in the latter's case, the cannot significantly say, e.g., "the past is now, on August 1, 2015, is two years longer (or older, except metaphorically) than it was on August 1, 2013", appears to show that, as it is ordinarily conceived, we cannot meaningfully speak of the past itself (and so, time itself) as having had a beginning. This conclusion can also be reached as follows. If it had any meaning, the sentence, "How long is the past at this moment?". (S), would mean: "How many seconds, minutes, hours, etc. have elapsed since the past began (hence, by implication, since time began)? Hence if, as appears to be the case, sentence $S$ is meaningless, $S^{\prime}$ too is meaningless.

\section{II}

The Past, the Present, the Future

\section{A. The Past}

In speaking of the past, we refer to a certain constantly lengthening hence variable stretch of time-1, measured relative to the present and the future. It embraces all moments, minutes, hours, etc. that have passed. Correlatively it refers to the constantly increasing, variable set of things that once existed but are no more. In saying that it is a constantly lengthening stretch of time-1 I do not mean or imply that it literally makes sense, at a given moment, to ask: "How long is the past 2 ?". Again, it literally makes no sense to ask how much longer it is at this moment than it was five, ten or twenty hours, etc. ago, given the passage of these hours, etc. For example it literally makes no sense to say: September 11, 2015 is two years longer than it was on July 11, 2015" though it clearly makes sense to speak of say "The Christian Era" as being today, on September 11, 2015,

Christian era, as being, on August 1, 2015, two years longer than it was on August 1, 2013. 
two years longer than it was on September, $2013^{5}$. The conclusion that it makes no sense to speak of "September 11, 2015 as two years longer than it was on September 11, 2013," can also be reached as follows: If it has a meaning at all, the sentence, "How long is the past at this moment?" would also have a meaning. But the idea of the past as having a beginning makes no sense, and so the question: "How long is the past now?" is meaningless hence lacks a meaningful answer.

The correct conclusion to draw from the preceding is I think that a category mistake is committed if we apply either (a) the concept of a beginning, or (b) the concept of absence of a beginning to the past ${ }^{6}$. (a) and (b) are shown by the fact that though we think of the past as a constantly lengthening period of time, what we mean is quite different from what we mean when we think of the present as a constantly changing stretch of time. Clearly the present can be literally said to be a stretch of time - a stretch of time since, as William James pointing out, the present is not a "knife-edge". As present, it exists while the past does not exist, or no longer exists. At any given moment in the present, the past ceases

5 Clearly, this "beginning," if it makes sense to speak of time-1 or the past in this way (but see above) cannot be a beginning in the usual sense, i.e., in time, but a beginning "relative to" a timeless eternity, whatever that may mean. This can also be seen as follows: Let us suppose that the past $_{2}$ did have a beginning. Then time $_{2}$ too had a beginning - just one moment 2 before the past $t_{2}$ began. For as soon as the first moment ${ }_{2}$ passed the past ${ }_{2}$ began! That is clearly meaningless, or absurd.

6 This is quite different from saying that the concept of time $_{1}$ ceases to apply to the singularity called the Big Bang, which is maintained by Albert Einstein and cosmologists such as Hawking and Grünbaum among others. In Hawking's cosmology, the concept of "real time" (as opposed to "imaginary time" (time 1 in my terminology) stops applying to the universe's beginning.

7 The same applies, mutatis mutandis, to the future ${ }_{2}$, since by definition, it does not yet exist. to exist. (Is 'exist' the right word to apply to the past?) What does not exist cannot possibly have a beginning or not have a beginning: to speak in that way would be to use words without meaning. As a consequence, it makes no sense to speak of the past as having, at a given present or past moment, a certain length; and the like ${ }^{7}$. But existing things such as objects, persons, events, etc., so long as they exist, can be said to exist for a certain length of time . $_{\text {. }}$

The discussion so far has left us with the following basic question: What are we to understand by "The past is a certain constantly lengthening stretch of time?". In light of our earlier discussion about the nature of past time, etc., I think the answer is that (a) the past consists of those moments of time 2 - or minutes, hours, etc. - that have passed, ceased to exist (hence were once present moments, minute, etc.); and that (b) the number of these moments, etc. is continually increasing.

\section{B. The Present \& the Future}

Turning to the present 2 , 'the present ${ }_{2}$ ' refers to a certain variable, constantly "shifting" - not lengthening or shortening - stretch of time. And by 'shifting' I mean that what we call 'the present" at different moments varies with what exists at that minute $_{2}$, hour, etc.

'The future' 2 too refers to a certain constantly "shifting", not lengthening or shortening, stretch of time. But the sense in which the future "shifts", is clearly different from the sense in which the present" "shifts". Indeed, the phrase

In Mysteries of Modern Physics: Time, his Course Guidebook to the DVDs of his lectures in The Great Courses, The Teaching Company, 2012, Prof. Sean Carroll of California Institute of Technology provides nice comparisons between the past and the future in "How Is the Past Different from the Future?", pp. $24 \mathrm{ff}$. 
'constantly shifting stretch of time' is figurative since the future by definition, does not yet exist, has not yet come to pass. What I have in mind is that some things that are in the future at any given moment constantly come into existence; ${ }^{8}$ and correlatively, that minutes 2 , hours 2 , days, etc. that at a given moment had not yet existed, constantly pass into existence, become present minutes, hours, etc. Compare with the uses of 'the past', 'the present', 'the future'. The latter expressions do not refer to anything that can be said to be constantly lengthening, or shifting, in any sense of these words.

(1) Unlike existing things, the past itself does not recede into anything, e.g., the more remote past 2 . The statement "An existing thing $E$ has receded into the past" $(\mathrm{P})$ presupposes, hence does not have the same meaning as, "The moment $_{2}$, 'hour' etc. in which $E$ existed has ceased to be". This illustrates the significant point that some temporal statements about things are not synonymous with or translatable into statements about (a) moments of time, minutes, hours, etc., (b) the past, present, or future, or (c) time itself (time $_{1}$ or time, depending on the particular object-statement). Thus "The present year (2015) is receding into the past" is different in meaning from (i) "All objects, persons, etc. that exist this year, in 2015, are receding into the past". Nor is it the same as (ii) "Some objects, persons, events, etc. that, at midnight, December 31, 2014, were in existence, have ceased to exist", or (iii) "Some objects, etc. that were in existence at midnight,

8 Note that we do not, at least not usually, speak of objects and persons as lying or being in the future, except perhaps in a figurative sense. More naturally, we speak of an unborn child's birth, a wedding, a graduation, or some other event or change as lying in the future.

9 We do not say that the present ${ }_{2}$ itself is receding into the past or has ceased to exist. Hence 'the present moment $_{2}$ ', is not always interchangeable with 'the present $_{2}$.
December 31, 2014 have ceased to exist". And so on. However, "The present year (2015) is receding into the past" entails (ii); and (iii) - hence (ii) - logically presupposes it.

As I mentioned earlier the past does not recede into anything; ${ }^{10}$ but we do say: "As the years pass, 2014 - last year - will recede further and further in the past, become more and more past". It would therefore seem that the past is not made up of past years, hence of (past) minutes hours, days, etc., - which is clearly false. To see this, we need to note the distinction between (a) the date, 2014, and, (b) the 364 days of which the year 2014 was composed. The word 'year' in ' 2015 ' is used in both senses: in (i) the sense of a certain date, a certain 'point' in time relative to other 'points' in time, in a particular year that comes to be then ceases to be; and (ii) in the sense of a certain length of time, i.e. a year. The year 2015 that recedes into the past is the year, and so is the 364 days of which that year consists and is gradually receding into the past.

(2) In its nature or as such, the ordinary concept of time ${ }^{11}$ has no reference itself whatever to, is totally independent of, any and all of the entities, processes, etc., that exist or takes place in time, beginning perhaps with the quantum fluctuations that many physicists/cosmologists believe somehow gave rise to the Big Bang. That is reflected, for example, in Isaac Newton's definition of "absolute" time: "Absolute, true and mathematical time, of itself and from its own nature flows equally without regard to anything external, and by another name is called dura-

10 Clearly this is also true, though in another sense of the past $_{1}$. For the concept of passage, hence of receding makes no sense in relation to 'the past 2 '.

11 In some respects what corresponds to time 2 while in other respects, I think, what corresponds to time . Note Newton's definition or description above. 
tion..." ${ }^{\prime 12}$ Compare and contrast this with the ordinary concept of time. It is clear that if nothing whatever existed, nothing could be said to exist for an hour, a day, etc., and nothing could be said to exist for an hour (to happen), a day, etc., and nothing could be said to take an hour (to happen). Further, if nothing existed - which means among other things, that no sentient beings had existed to utilise certain happenings to demarcate "the present" and so, together with other data. "The past" and "the future" - the concepts of past, present, and future - as well as those of past, present and future - would not have had any actual application. In short, the concept of time as a whole would have no possible application; since these concepts are essential parts of the general concept of time. But as I wrote in "On Time," "we...cannot [ordinarily] say, on the basis of the ordinary meaning of 'time' [or the concept of time] whether or not there may or would be time if the universe had not existed at all” (Khatchadourian, 1961, p. 464). To assert or to deny that time could and would exist if no universe (including God) did exist, would be to apply temporal expressions to a "state of affairs" to which they are not, in the ordinary concept of time, intended to apply; with respect to which ordinary language is silent ${ }^{13}$. Contrast this with the state of affairs in which the universe, as we know it, is conceived as having ceased to exist (Khatchadourian, 1961, pp. 464f., 466).

Further, time is not a kind of entity. To show that, we would need to examine much more closely than can do here the ordinary uses

12 Mathematical Principles of Natural Philosophy, p. 6. Quoted from (Nagel, 1961). But here Newton confuses the concept of time as a whole and the concept of the passage of time it includes.

13 Contemporary physicists/cosmologists believe that time began with the universe's coming to be: in the case of some, that time-zero started before Planck time -43 . of 'time'. I might just mention two possible indications that time in its ordinary conception is not some kind of entity. The first is that passage of time, a fortiori time itself, is not causally efficacious. One thing is clear. Time, as ordinarily conceived is not and cannot be a physical entity.

Finally, the foregoing facts (or what appear to me to be facts) about the ordinary concept of time provide additional evidence that the passage of time cannot be a form of change; since a change that cannot possibly lead to or result in further change would be an extremely odd kind of change. The same is true of a change that we cannot meaningfully say has - or does not have a beginning!

(3) The foregoing (2) indicates that the ordinary concept of time is not identical with the absolutist conception of time; though the contrary is, I think, commonly believed by philosophers. But there are some similarities between the two, which are worth noting.

(a) Time is not any kind of relation between events, or between event, objects, living organisms, and other kinds of things that as we ordinarily say exist or occur. Indeed, not only is "time 1 " not a kind of relation on the ordinary conception of "relation" (see Khatchadourian, 1964): temporal expressions such as 'before,' 'after' and 'simultaneous with' do not designate any relation(s) in any ordinary meaning of 'relation'. Consequently, to speak of the passing moments of time, or of seconds, minutes, etc. as related to one another insofar as they succeed one another, is to use the word 'relation' in a non-ordinary way.

(b) Related to (a) above is the fact that on both conceptions of time, temporal concepts are logically independent of the concept of change. Indeed, both concepts imply that 
the existence of time a logical condition of the very possibility of change in the universe $^{14}$.

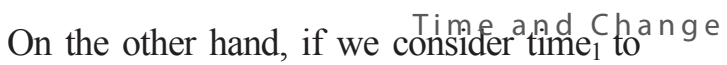
be an a priori logical construct - that is, if it is not some sort of "objective reality" - time 1 would clearly not have "existed" if we did not exist; and that if we cease to exist, time ${ }_{1}$ would likewise cease to "exist". But on the absolutist conception time is an objective feature of reality itself, not a logical construct. Hence it would, or could, exist whether or not we ever existed, and would, or could, continue to exist when we are wiped off the face of the earth. (The same, mutatis mutandis, applies to possible humanoids inhabiting other planets in the universe. It would be interesting to speculate that such beings may perhaps have very different temporal concepts from ours to organize their experiences. Or would that be impossible? In other words, is some conception of time, bearing a fairly close similarity to the ordinary, the absolutist, the relativity or the relational conception of time, inescapable if we (or possible humanoids) are to make sense of our (or their) experience ${ }^{15}$ ?

14 However, if change did not exist, we presumably could not have formed temporal concepts and structured our experiences in terms of time. (See (Khatchadourian, 1961), passim.)

15 Immanuel Kant's "subjective" view on this general subject is familiar. But does, or would, empirical evidence support Kant's view? That, and the above, are some fundamental questions a philosophy of time must come to grips with. But this is not the place even to begin to try to answer any of them.

See (Khatchadourian, 1961) passim, for a critique of Henri Bergson's claim about the existence of a variable "subjective time" - a kind of time distinct form "objective time," varying with our inner experiences. Note the following common imaginative, metaphorical way of speaking of time's passage, quoted from Ivan Turgenev's Fathers and Sons: "Time, it is well known, sometimes flies like a bird, sometimes crawls like a worm; but man is wont to be particularly happen when he does not even notice whether it passes quickly or slowly." (translated by Constance Garnett. The Modern Library, N.Y., [n.d.], p. 103.)
A third similarity is that on the ordinary conception, time literally passes regularly, not sometimes "slowly" and sometimes "quickly". Or more precisely - since in the ordinary concept time's slowness or quickness are literally only applicable to the entities, events, etc., that exist in time at any given moment, hours, day, year, etc., not to time itself, any one moment of time is as long or as short as any other moment ${ }^{16}$.

III

\section{Time; Some Current Cosmological Conceptions/Speculations ${ }^{17}$}

(1)(a) Einstein's conception of time in his theory of General Relativity: conjoining time with space in a four-dimensional continuum of space-time; and, the relativity hence variability of the passage of time depending on the observer's space-time position and velocity in the universe. That conjoining of space and time in space-time in a variably curved universe may have, as far as, as a layman, I can see, does not affect any fundamental changes in, does not modify, the ordinary concept of time itself; though I may be quite wrong and it in fact does effect an important, perhaps, profound, modification or change in the ordinary concept.

(b) Einstein's theory radically changes the concept of the passage of time ordinarily thought of as constant, not sometimes passing faster and sometimes slower, independent of where in the universe and the velocity with which he or she is moving the observer happens to be in the universe. On his theory, the passage of time slow down as the velocity of the observer increases, making the passage of time relative to the ob-

16 See (Taylor, 1963, Chapter 6, pp. 70-83). The Puzzles are in pp. 74-83.

17 As opposed to mathematical "abstract time". 
server's position and speed. A contemporary example would be the lapse of time in the case of astronauts flying to the international space station, or for the astronauts stationed there.

The conjoining of space and time in space-time in a variably curved universe may have brought about an important, even fundamental, change in the ordinary concept of time itself.

\section{The Arrow of Time}

The text that accompanies the DVD lecture series entitled Mysteries of Modern Physics: Time, Sean Carroll of California Institute of Technology states the following:

- "We actually define the past versus the future using the arrow of time" (Carroll, 2012, p. 27). Carroll, $S$.

- And importantly: "Time could exist in a universe without an arrow, and time is not the arrow itself. The arrow is a feature of the stuff of the universe... Things... evolve in certain ways always in the same direction, from the past to the future." And, "The arrow of time is the arrow of stuff evolving in time. ... It is not time that we need to understand but matter. It is the motion of particles and objects in the universe" 18 (Carroll, 2012, p. 28).

Question: Why and precisely how does the arrow of time, hence the passage of time depends on entropy? (So, if there was no change in entropy in the universe, time would have "stood still", hence would have ceased to exist?)

\section{The Second Law of Thermodynamics}

"...The feature of matter that changes with time is called entropy. The feature of the universe...is that the increase of entropy is associated with the passage of time".

"The second law of thermodynamics entropy of the universe increases - underlies all the ways in which the past is different from the future". "...[T]he fact that entropy increases is the reason you remember the past and not the future." "We usually find that when the entropy decreases in one small system, it is because the universe was increasing in entropy greatly in the universe" (Carroll, 2012, p. 28).

"But we're saying that the fact that entropy increases is the reason you remember the past and not the future" (Carroll, 2012, p. 28).

And, "The second law ... says that there is an irreversibility - a direction - of time. Entropy increases in one direction and decreases as we go to the past" (Carroll, "Time", p. 31). Carroll adds that the [previous] statement of it is actually only an approximation. "It is not absolutely impossible for entropy to decrease spontaneously; it is, however, extremely unlikely". "The second law is a metalaw; it refers to how different kinds of laws of physics can possibly work" (Carroll, 2012, p. 31). Further, “...entropy measures disorderliness. ... [A]nother way to think about entropy is as a measure of the uselessness of a certain amount of energy" (Carroll, 2012, p. 34).

"Energy is conserved, but it can change forms. If you have energy in a low entropy form, you can do useful work with it. ... If you convert that energy into a high-entropy form, it becomes useless. ... Fuel is a low-entropy concentration of energy" (Carroll, 2012, p. 35). 


\section{Time's Arrow ${ }^{19}$}

In "Time's Arrow" Carroll notes, inter alia, the following general points about Time's Arrow: "The... very strong arrow of time" the universe has, "which is reflected in the life cycle of stars and planets". And: "Besides physical and biological change, there are some features of the arrow of time that seem ingrained or logically necessary." That includes “...the idea that the cause will always precede an effect. ..." And: "A universe without the arrow of time... would not have progress or differentiation from the past to the future" (Carroll, 2012, pp. 24-26).

\section{Irreversibility of Time's Arrow}

- "Something happens in one direction, and it is easy to make it happen, but it does not happen in the other direction, or if it does, it is because we put effort into it. ... They go in the direction of time".

- "That difference between going from the past to the future is consistent throughout the universe as far as we know. This is... a

19 Throughout this section 'time' refers to what scientists/cosmologists call "real time" to distinguish it from "imaginary time", Hawking describes as "a welldefined mathematical concept" (see Hawking, 2009, p. 87). And, "The singularity theorems of classical general relativity showed that the universe must have a beginning, and that this beginning must be described in terms of quantum theory. This in turn led to the idea that the universe could be finite in imaginary time, but without boundaries or singularities. ... This might suggest that the so-called imaginary time is really the fundamental time, and that what we call real time is something we create just in our minds. In real time, the universe has a beginning and an end at singularities that form a boundary to space-time and at which the laws of physics break down. But in imaginary time, there are no singularities or boundaries. So maybe what we call imaginary time is really more basic, and what we call real time is just an idea that we invent to help us describe what we think the universe is like. ... Which is real, "real" or "imaginary" time? It is simply a matter of which is a more useful description" (Hawking, 2009, p. 91). feature of the way the universe works"

(Carroll, 2012, pp. 24-26).

Re Time in Stephen Hawking and Penrose:

1. The entropy of the universe must be progressively increasing while the universe undergoes a "Big Crunch", gradually returning to a new singularity, if Hawking is right that the Big Crunch would not result in a reversal of time's arrow.

2. Re Penrose's Cycles of Time. If a new cycle of time begins with every new cycle of the universe resulting not, as in the original cycle from [the] Big Bang from the white energy escaping from black holes (Hawking), then the entropy in each cycle must, like our present "time cycle," start with the lowest possible entropy and continually increase as the cycle evolves, for the arrow of time to continually increase from the past to the present and future; i.e., for it not to be reversed in any Cycle.

But how is it that the entropy will not gradually decrease, as according to Hawking, the Great Crunch begins and continues until a new universe starts from a new Big Bang? For the entropy of the mass and energy in the universe in the Big Crunch would seem to become gradually less.

\section{Stephen Hawking on Time's Arrow;}

The importance of time's arrow always pointing from the past to the future, never reversing direction, is well illustrated in Stephen Hawking's speculations concerning the "Big Crunch" in his book Black Holes and Baby Universes and other Essays (Hawking, 1994, pp. 146ff). For instance he writes: 
One would expect the gravitational attraction between different galaxies to be

[in future] slowing down the rate

of expansion. If the density of the universe is greater than a certain critical value, gravitational attraction will eventually stop the expansion and make the universe start to contract again. The universe would collapse to a big crunch. This would be rather like the big bang that began the universe. The big crunch would be what is called a singularity, a state of infinite density at which the laws of physics would break down. This means that even if there were events after the big crunch, what happened at them could not be predicted (Hawking, 1994, p 146).

"If [the density of the universe] is greater [than the critical value]. The universe will recollapse and time itself will come to an end at the big bang crunch $^{20}$ (Hawking, 1994, p 147). And about the idea of "time-travel" he writes: "What seems to happen is that the effects of the uncertainty principle would cause there to be a large amount of radiation if one travelled into the past [i.e. if the arrow of time were reversed]. This radiation would either warp space-time so much that it would not be possible to go back in time, or it would cause space-time to come to an end in a singularity like the big bang and the big crunch" (Hawking, 1994, p 154).

Hawking does not directly answer the question whether the arrow of time would be reversed during the big crunch. But the answer I believe would be "no," since the entropy of the universe during the big crunch would presumably not decrease or may perhaps even continue to increase.

20 My italics.

\section{Roger Penrose on Entropy ${ }^{21}$}

In the 1879s Ludwig Boltzmann gave us "a more applicable definition of entropy than that given earlier by Rudolf Clausius. In Boltzmann's definition, "heat is...thermal energy the random motions of atoms. ... Boltzmann realized that arrangements of atoms are macroscopically indistinguishable, and... that entropy is simply a way of counting the number of arrangements of atoms inside a certain system". "In other words, the reason entropy increases, according to Boltzmann, is simply that there are more way to be high entropy than to be low entropy. That is a rigorous definition that corresponds to our intuitive feeling that entropy measures disorderliness" (Penrose, 2012, p. $36)^{22}$.

"When entropy is low, the macroscopic configuration is very precisely arranged. There are only a few such configurations that look the same. When entropy is high, the configuration is spread out. There are many different ways to arrange the atoms, and all of them look alike". "Boltzmann's definition of entropy is one that makes the arrow of time go. Once we understand it, we can ask why entropy was so low in the early universe" (Penrose, 2012, p. 36) ${ }^{23}$.

Penrose devotes Part I, sections 1.1-1.5 to entropy: "The relentless march of randomness", (Section 1.1); section 1.2 to "Entropy, as state counting”, 1.3 largely to Boltzmann's definition of entropy; 1.4 to the "robustness of the concept of entropy"; and 1.5 to "The inexorable increase of entropy into the future"; and 1.6 to "Why is the past different?". For instance, in 1.4 he pro-

21 See (Penrose, 2012, pp. 11ff).

22 Here refer to Penrose's more rigorous definition of entropy in Cycles of Time.

23 Later we will consider the question "why entropy was so low in the early universe". 
vides a more precise definition of entropy than Boltzmann's definition ${ }^{24}$.

The reason(s) why the past is different from the future, Penrose says is that, argues against the idea that there is no mystery for our experience of the passage of time as "dependent upon an increasing entropy as part of what constitutes our conscious feeling of the passage of time; so whatever time-direction we believe to be the 'future' must be that in which entropy increases. He argues that "this argument misses the crucial point that the very usefulness of the notion of entropy depends upon our universe being enormously far from thermal equilibrium, so that coarse-graining regions that are far smaller than $R_{\max }$ are involved in our common experience. In addition to this, the very fact that entropy is either uniformly increasing or uniformly decreasing depends upon the actuality of one or the other end (but not both ends) of the evolution curve in phase space being constrained to a very tiny coarse-graining region, and this is the case of only a very minute fraction of possible universe histories. It is the very tininess of the coarse-graining region ' $B$ that our evolution curve appears to have encountered that needs explaining, and this issue is completely untouched by the aforementioned argument" (Penrose, 2012, p. 3).

\section{Why is the Past Different?}

"The answer to that question [he writes], 'though hardly a 'physical explanation' - is

24 Using natural logarithms, he writes 'Bolztmann's entropy formula as: $\mathrm{S}=\mathrm{k} \log \mathrm{V}$, where $\log \mathrm{V}=$ 2.302585 .. x $\log _{10}$ V" (Penrose, 2012, p. 30).

Later Penrose adds about entropy: “...we may well take the view that the Second Law is proceeding according to its normal practice, where the entropy continues to increase - but we must be careful about what 'entropy' notion we are referring to here. This entropy refers to all the degrees of freedom, including that of all the material that has fallen into the [black] holes" (Penrose, 2012, p. 188). simply that such 'past-teleology' is common experience, whereas 'future teleology' is just something that we never seem to encounter... It is...observational fact that the Second Law holds good. In the universe we know, the dynamical laws appear not to be guided in any way to a future goal and can be regarded as being completely unconcerned with coarse-graining regions; whereas such 'guidance' of the evolution curve in past directions is utterly commonplace. ...such 'teleology' is perfectly acceptable if we are looking towards the past, but it is not a feature of our experience that it apply towards the future" (Penrose, 2012, p. 51).

And ... The very origin of our universe was represented in phase space by a course-graining region of quite exceptional tininess, so that the initial state of the universe was one of particularly small entropy. ... The Big Bang had, for some reason, an extraordinarily tiny entropy... So, the key issue is indeed the specialness of the Big Bang, and the extraordinary minuteness of the initial course-graining region ' $B$ that represents the nature of this special initial state" (Penrose, 2012, p. 51).

(2) According to contemporary physics and cosmology, ${ }^{25}$ real time (which is contrasted with mathematical, imaginary time) began with the quantum fluctuations that some contemporary scientists believe resulted in the Big Bang. Given that over 15 billion years have so far elapsed since the beginning of real time.

Concerning the ultimate origin of the universe before the Big Bang, hence about the beginning to real time, "three current speculations" (Trefil, 1985, p. 238) consist in (a) The Geometrical Approach, propounded by Francois Englert

25 Who? E.g. is this Hawking's or Penrose's view? And what about the cosmological theories of the origin of the universe? 
and Raoul Brout", (b) The Multi-Dimensional Approach, and (c) The Dynamical Theories. These speculations speculate about the time before ${ }^{-43}$ Planck time, which is "the earliest instant that present theories can address" (Trefil, 1985, p. 240). These speculations "have one thing in common: they all agree that somewhere around the Planck time something radically different happened, and that the concept of time zero has no more meaning for the universe... (Trefil, 1985 , p. 240$)^{26}$

The geometrical picture provides a "picture in which the universe was an ordinary vacuum at a time long before ... the start of the Big Bang ... [T] he vacuum is inherently unstable ... If a small bit of matter should appear ... its effect would be to make the grid expand slightly". "The positive energy need to create the bit of intrusive matter is balanced by negative energy stored in the expanding grid, so that there is no violation of the [second law] of the conservation of energy involved in this sort of event" (Trefil, 1985 , p. 240). "... Once the grid starts to expand at any point, more matter is created". And "so on", "until the universe reaches the proportions it had at the Planck time" (Trefil, 1985, p. 241). "As in the case in most of the other theories... the geometrical case regards the initial bit of mass as the product of a random fluctuation of the type often seen in subatomic systems. It triggers the instability of the grid". "The potential for growth [until it grows] "into the present universe. The potential for growth is inherent in the dynamics of the vacuum" (Trefil, 1985, p. 243).

The third, Dynamical theories, Edward Tryon (and others) speculate that "A particle in empty space, provided that the opposing particles annihilate each other in a time so short that

26 My italics. the violation of energy conservation implicit in the process cannot be detected. Particle-antiparticle pairs pop into existence here and there but disappear quickly" (Trefil, 1985, pp. 241-242). "In this [Edward Tryon] picture, the universe came into existence as a fluctuation in the quantum mechanics vacuum - a notion that leads to a view of creation in which the entire universe is simply an accident" (Trefil, 1985, p. 242).

"A more modern version" is described by David Schramm:

In this scheme of things, if we could see the universe before the Planck time, we would find the elemental foam extending into the infinite past. As in the vacuum fluctuation picture, the universe began when enough "bubbles" happened by chance, to come together. Unlike the fluctuation picture, however, in this scheme the Big Bang actually started at the Planck time. There is no time zero and hence no singularity (Trefil, 1985, p. 242) ${ }^{27}$.

Trefil comments about the three types of speculations thus:

Implicit in all of our discussion of what may have happened before the Planck time is a very singular idea. It holds that the laws we can discover in our laboratories here and now have governed not only the universe since the beginning, but governed the moment of creation as well.... We are saying that the very existence of the universe is an inevitable result of the laws of physics (Trefil, 1985, p. 243).

\footnotetext{
${ }^{27}$ My italics. The idea that at the Big Bang the laws of physics break down, hence a singularity, comes from Einstein's General Theory of Relativity.
} 
VII. Additional Questions or Issues Regarding real Time in Relation to Certain of (a) Hawking's, and (b) Penrose's Cosmological Theories.

\section{Real vs. Imaginary time.}

1. Re Carroll: Why and precisely how does the passage of time depend on entropy? The answer must be that as entropy increases, time passes, time's arrow flies from the past to the future. What do Carroll and/or Penrose say about it - if anything? If, for some known or unknown reason there were no change in the universe's entropy, time, according to Carroll, would stop, cease to exist.

2. Re Penrose: If a new cycle of time begins with every new Cycle of the universe and results not, as in the original Cycle from the Big Bang but from while energy is escaping from black holes (Hawking), then the entropy in each Cycle must start - like our present Time Cycle with the lowest possible entropy and continually increase as the Cycle evolves, for the arrow of time to continually point from the past toward the future; i.e., for it not to be reversed in every Cycle.

It is clear that the first Cycle of time could only have come from a Big Bang and could not have come about from a white hole. Only subsequent "big bangs" could come from already existing black holes - which implies that the succeeding Cycles can only come from already existing universes or "baby universes" (to borrow the phrase from Hawking) left over from a previous, incomplete Cycle - i.e., a Crunch that occurred only partially and not completely, i.e., not going all the way to the very beginning of the universe.
A further problem concerning Penrose's view of an aeon preceding the Big Bang of our aeon. Presumably, such an aeon could only arise from a previous Big Bang of our aeon, not from a White Hole (the evaporation from a huge black hole) since, presumably, no black holes would exist without the existence of galaxies with black holes at their centers, hence a massive black hole could not have originated the previous aeon.

- As long as matter and/or energy exist in the universe, there would be time.

- Since the arrow of time always points from the past toward the future, determined by the universe's increasing entropy, the arrow will always point in the same direction.

- The ordinary uses of 'time' vs. the scientific concept of "real time".

- If nothing existed instead of a universe, there would have been no time (or space), since complete nonexistence of matter or energy would have meant the total absence of entropy, zero entropy. With the presumed quantum fluctuations - if these were actually the beginning of something existing - there would have been some incredibly low entropy (which is what Carroll states about the beginning of the universe) hence time - and its arrow - pointing from the lowest entropy to the progressively increasing entropy as the universe evolves, the arrow of time will continue to move in the same direction, from past to future, just as we know.

Why and precisely how does the passage of the arrow of time depend on entropy? So, if there were no change at all in entropy in the universe time would stand still, hence would cease to exist? 


\section{IV}

Time and Change

It may be recalled that two of the claims I made before are: (1) that the passage of time is not a form of change, and, correspondingly, (2) that the being or "enduring" of entities, events, occurrences, etc., in time is not a form of change, undergone by them. In this section I shall endeavour to show how a considerable number of confusions, puzzles or paradoxes that would result from one's supposing that the opposite of (1) and (2) is true. To that end, I shall consider the puzzles or paradoxes with which Richard Taylor, in "Time and Becoming" (Taylor, 1963), is confronted with maintaining that the passage of time is a form of change. The discussion should add to what I said about time and temporal passage and illustrate a number of the points I made.

Taylor starts his consideration of "temporal passage and becoming" inauspiciously by supposing, as I said, that the passage of time is a form of change (Taylor, 1963, p. 70). He is misled by the fact that we use the term 'passage' (also 'passing') in speaking of time, and using 'drawing nearer', 'becoming present', and 'receding', in speaking of things that endure in time. He writes:

\begin{abstract}
The one thing about time that has always been the greatest stumbling block to comparing it with space... is its passage or flow ${ }^{28}$ or, what amounts
\end{abstract}

28 Note the metaphorical 'flow' Taylor adds, in line with a long poetic tradition, reinforcing his error about the passage of time. to the same thing, ${ }^{29}$ the characteristic all things seem to have of continuously moving through time. Thus we speak of future things as drawing nearer, of then becoming present and, having passed into the present, of receding endlessly into an ever-growing past. Such expressions as these imply that something is moving, though they do not imply that anything is moving in space, in the usual way things move. In fact, a thing needs only to have a place in time in order to be moving in the manner suggested; for concerning any object that ever exists in time we can say that, until it exists, it draws closer to existing ${ }^{30}$; that while it exists it becomes older; and after it ceases to exist it recedes ever farther into the past (Taylor, 1963, p. 74) ${ }^{31}$.

And later:

It [the passage of things through time] is aptly called pure becoming because any other kind of change or becoming that anything might undergo presupposes this kind of change, whereas this pure becoming presupposes no other change at all (Taylor, 1963, p. 76) . $^{32}$

29 Taylor is implying, without any semantic evidence, that time "would not exist" (or that, perhaps, that it would not make sense to speak of the passage of time and hence of time itself) in the absence of things that endure in it. Compare in (Khatchadourian, 1961).

30 This last sentence is fraught with strange metaphysical presuppositions, which can be seen e.g., by asking how something that does not yet exist can "draw closer" to anything, including existing; except in the obvious ordinary sense that the time or date of its coming into existence is drawing nearer with the passage of time. This is independent of the question whether whatever will come into existence is causally determined to come into existence.

31 Italics in original, except for 'moving,' which is my italics.

32 Italics in original. 
It is not at all surprising that the passage of time or the existence of objects, events, etc., in time, thought of in this way, has "always profoundly bewildered philosophers, because it seems to be loaded with absurdities as soon as one begins thinking about it" (Taylor, 1963, p. 74-75). Strangely but not uncharacteristically however, philosophers, Taylor included, have failed to draw from this fact the obvious conclusion that the generation of these absurdities is really a reductio ad absurdum showing in no uncertain terms the existence of a serious error in this case a category mistake $e^{33}-$ in at least one of the premises of this inference: the premise that the passage of time, or the enduring of things in time, is a form of change ("becoming"). If this had been detected, philosophers would not have had to resort to the Draconian measures to which, Taylor points out, some have been forced to resort: the denial of the reality of the passage of time, hence the reality of time itself (whatever that may mean, or whatever it may have meant in each particular case) ${ }^{34}$.

I should emphasize that to maintain that the concept of change cannot be meaningfully applied either to time in general or specifically to the passage of time, is not to deny that time passes, and certainly not to deny that time is in some sense "real". I say this because Taylor writes: "Confronted with such considerations as these [the paradoxes of "pure becoming"] it is tempting to dismiss pure becoming or temporal passage of things is an illusion, to say that nothing ever really does change in this sense" (Taylor,

33 Cf. (Ryle, 1945) and (Khatchadourian, 1967, chapter 8).

34 Cf. (Taylor, 1963, p. 75). However, I do not think that Taylor is right in holding that the idea "that time itself is unreal, and that the passage of things through time is an illusion, has been almost characteristic of metaphysics ever since [some of the earliest metaphysical speculations of the Greeks]" (Taylor, 1963, p. 75).
1963, p. 78). Taylor here and everywhere else in his article supposes that it is proper to say that "The passage of time is a form of change", or "There is pure becoming", hence that these statements are true or false. (Of course, he believes that it is true.) Consequently, he believes that to deny "pure becoming" is tantamount to denying that anything exists in time, or that time passes. And according to him, that would amount to "denying the datum with which we [Taylor] began". He continues: "Moreover, in case the idea of the passage or flow of time is essential to the very conception of time itself, as it may well be, then to deny that there is any such passage would amount to denying that time is real, or that anything is even in time at all" (Taylor, 1963, p. 79).

What I do deny is not the 'reality' of time or its passage; and I fully agree with Taylor that to deny the passage of time is to deny the 'reality' of time in some sense of 'reality' or other. That follows from what I said earlier about the relation between the uses of 'time' and the passage of time. Taylor's view and what he says about the passage of time, etc., show that he thinks 'passage' and 'flow' have the same kind of use or meaning, ${ }^{35}$ in this context, as in relation to things (objects) that move in space, or things (liquids) that flow; and so on. For example he says: "...A thing needs only to have a place in time in order to be moving in the manner suggested; for concerning any object that ever exists in time we can say that, until it exists, it draws closer and closer to existing; that while it exists it becomes older; and after it ceases to exist it recedes ever farther into the past" (Taylor, 1963, p. 74). Perhaps his earlier emphasis on the similarities between space and time (see Taylor,

35 I do not say: "same meaning or use" because Taylor does point out that the passage in question here is not passage in space. 
1963 , p. 70-74) is a factor in his assimilation of 'passage,' 'flow,' 'recede,' and the like in their everyday uses, in relation to objects moving in space. Taylor mentions several strange consequences of the view he advocates. (1) The first of these is that:

Even things which have ceased to exist, and others which will exist in the future but do not exist yet, undergo a relational kind of change which is simply a corollary of pure becoming or passage through time. That is, we can speak of Diogenes' cup as receding even farther into the past. It is more remote from us in time today than it was yesterday, and this is a relational change which it is undergoing. Similarly, the birth of my first grandchild, assuming there will be one, is something that is drawing closer and this is a relational change that something which will, but does not yet exist, is already undergoing ${ }^{36}$ (Taylor, 1963, p. 77).

\section{Two comments are in order.}

(1) Diogenes' cup, which does not exist anymore, cannot in any literal sense recede, and so recede farther, into the past. Similarly, mutatis mutandis, the "birth of my first grandchild" cannot be "drawing closer". This is true whether or not the passage of time is a form of change, or a relational form of change; and whether or not "receding" and "drawing slower" in the present context should be interpreted as a form of change. What is ordinarily meant by saying that Diogenes' cup is receding farther into the past is, as we saw before, (a) that the dates on which it existed have passed, and (i) that these dates are receding father into the past, (b) itself

\footnotetext{
36 Italics in original.
}

means (i') that an ever-increasing number of moments $_{2}$, hours 2 , etc., have been succeeding the minutes, days, etc., in which it existed. The same sort of explication is possible, mutatis mutandis, with respect to the statement that the birth of my first grandchild is drawing closer.

In light of (1) above it is seen that only the duration ("enduring") of existing or present 2 things, while they exist ${ }_{2}$, can at best be meaningfully said to be a form of change ${ }^{37}$. Yet even that will not do, for the same basic reasons I gave earlier, and those I shall give under (2) (5) in this section, against the view that the passage of time is a form of change. For only if the latter is a form of change can the duration of existing things in time ${ }_{1}$ be a form of change; the change which I for instance am supposed to undergo qua "enduring" in ( present $_{1}$ ) time can only be the change in which the passage of time allegedly consists. (Here one may ask how the latter, not being causally efficacious, can produce any change in me.)

(2) Taylor states the second basic puzzle as follows:

It seems, moreover, that times themselves undergo both kinds of

37 Note that the passage of time ${ }_{2}$ is logically an ultimate, unanalysable concept which we logically end up with whenever we talk about the existence of objects, persons, events, etc. - of things that exist in time. That is a part of the "fact" that the concept of time ${ }_{1}$ and the concept of time ${ }_{2}$ form part of the logically basic conceptual framework of ordinary English, French, German and perhaps many other, if not all ordinary languages.

With respect to Taylor's view that Diogenes' non-existent cup is undergoing a relational kind of change, I might point out that in my (Khatchadourian, 1973). I argued that the succession of moments ${ }_{2}$, etc. does not involve a relation between moments $t_{1}$ and $t_{2}$ - a moment $\left(\mathrm{t}_{2}\right)$ that is succeeding or has succeeded another moment $t_{1}$. Hence the puzzle as to how an "existing" moment $\left(\mathrm{t}_{2}\right)$ can be related to something that no longer exists $\left(t_{1}\right)$, and to something that does not yet exist $\left(t_{3}\right)-$ the moment that will immediately follow $t_{2}-$ does not arise. 
change just described. Any actual time,

like any actual being, undergoes the same pure becoming or passage as the latter, and any nonactual past or future time undergoes the same relational change characteristic of non-actual past or future beings. Thus I can say of today, which is an actual time, that it is passing, elapsing, its end drawing closer, that it is becoming older (Taylor, 1963, p. 77).

If we recognize that the passage of time $_{2}$ is not a kind or form of change the foregoing difficulties do not arise. For (a) the utterly strange if not preposterous idea that "any nonactual past or future time [which does not exist] undergoes the same relational change characteristic of nonactual past or future beings" will not arise; just as I pointed out under (1) above, the idea of non-existent beings or things undergoing a "relational change" will not arise. Similarly with the difficulty that "It is exceedingly odd, then [in view of the fact that "all ordinary changes, or instances of things gaining or losing properties, presuppose time"] to suppose that times themselves should ever be thought of as changing, unless one is willing to assume a mysterious kind of metaphysical "time" against whose background the ordinary time intervals into which we are born and grow older can themselves come into being and age" (Taylor, 1963, p. 78$)^{38}$.

Taylor might have added that, on pain of having to posit an endless array of "metaphysical times", the "mysterious metaphysical time" to which he refers in the preceding quotation must be considered either (i) as not passing, ${ }^{39}$ or

38 Cf. (Khatchadourian, 1961, p. 464).

39 This time that is not passing cannot, however, be time or anything like time ${ }_{1}$ (see above); and the arbitrari- (ii) as a time whose passage is not and cannot be a form of change. Since on the ordinary conception of time (also, the other conceptions that have been formulated in Western thought at least, such as the Newtonian and the Relativity conceptions) it is self-contradictory to think of time (2) as not passing, we can see how in this conception, 'time 1 ' cannot refer to any kind of entity or thing, in which moments of time 2 are supposed to pass. For the ordinary concept of the passage of moments of time does not logically necessitate the supposition that "time, 1 " is something in which this passage takes place for the simple reason that the passage is not a form of change. Time is not something, in which moments of time 2 pass, metaphorically not unlike a river-bed in which flows a body of water composed of innumerable drops. In this way the ordinary concept of time avoids the difficulties that plague the conception of the passage of time ${ }_{2}$ conceived of as a form of change, hence the conception of time ${ }_{1}$ as some kind of entity, some kind of "receptacle" (which, however, does not undergo any kind or form of change, including the form of "change" the passage of moments of time is, on this view, supposed to be). Taylor fails to see these things because, once again, he misconstrues what we mean in speaking of the passage of time (Taylor, 1963, p. 78). Likewise he misconstrues "receding into the past" as some kind of process, happening or event. That is precisely involved in his thinking of, e.g., "today" or "tomorrow", in passing, as itself undergoing change.

(3) A further problem in Taylor's view we are considering stems from a fact I have pointed out, namely, that the notion of rate of change is inapplicable to the passage of time, hence to the

ness of positing a time that is so unlike what we call the (time or time $_{2}$ ) is, I think, quite evident. 
enduring of things in time. Interestingly, Taylor recognizes this, for he writes: “...The continual recession of things past, and the advance upon us of things future, is a strange kind of passage for the reason that no rate of passage can possibly be assigned to it without making nonsense" (Taylor, 1963, p. 78) ${ }^{40}$. This difficulty too is eliminated once the passage of time, or the enduring of things in time, is not supposed to be a form of change.

(4) Taylor rightly points out that " $\ldots$ it is... quite odd to think of events as themselves changing" (Taylor, 1963, p. 78). The reason is that the notion of change is inapplicable to an event, which is something that occurs, just as a change is something that occurs. Thus, it is meaningless to speak of change (or of a change), or of an event, as itself changing or not changing. A change begins and comes to an end; but beginning and coming to an end are not themselves change of any kind; they are not a change in anything or into anything: while all change, in the ordinary uses of 'change,' is of the one or the other sort ${ }^{41}$. The same is true, mutatis mutandis, of events. The upshot is that it would be utterly confused to speak of an event's being in time as a form of change. Yet Taylor persists in thinking that that view is apparently inescapable, since "... events are in time and have an endless recession into the past, like anything else" (Taylor, 1963, p. 78).

(5) Finally, Taylor points out that:

Pure becoming is not only an unobservable change but is compatible with, and in fact entailed by, any change whatever that is observed. If, for instance, we see

40 My italics.

41 I am not, of course, talking about the sort of change that we call "change of mind" or "change of heart," etc. that something, such as a leaf, is green and then becomes red, or red and then becomes green, we must conclude that it also becomes older, for nothing can become anything at all without becoming either older in the process.

The conception of something's becoming older, then, is a purely a priori notion, a consequence of its simply being in time (Taylor, 1963, p. 78).

If "pure becoming" - the passage of time is unobservable, in the sense of being unobservable in principle, which is what Taylor appears to imply - it would certainly constitute a further difficulty for Taylor's view. For a "change" that is unobservable in principle must be a very odd kind of change. To deal adequately with this point, however, one must first determine whether, and in what sense, the passage of time is or is not observable in principle, irrespective of any theories we may have regarding the nature of this passage. And that would require much more space that we have. Still, I might mention that if it can be shown that the passage of time would be unobservable in principle if we suppose that it is a form of change that would provide further grounds for maintaining that the passage of time is not a form of change. For there is, most assuredly, an ordinary sense in which we speak, at different times or in different situations, of having a "sense of the passage of time", and of "losing all sense of the passage of time". And we distinguish this "sense of (the passage of) time" from our purely conceptual or intellectual knowledge that time is constantly passing. On the other hand, there is a serious question as to whether there is such a thing as awareness of the passage of time as something distinct from awareness of change. In any case, if there is an "awareness of 
the passage of time", we are I think on fairly safe ground in supposing that we are aware of the passage of time, whatever that may mean, only through our awareness or observation of change in the broad sense $\mathrm{e}^{42}$.

Taylor attempts to overcome the foregoing difficulties without abandoning his original "datum" that the passage of time is a form of change. If we agree that the passage of time is not a form of change, we would expect that his attempt will inevitably fail. And that is precisely what happens on Taylor's own admission. For after several pages of closely reasoned analysis, Taylor ends up the chapter with the following admission: "We seem justified in concluding, then, that the pure becoming of the world and of time [translate this into: "the enduring of things in time and the passage of time] is no myth or illusion, however obscure it may be to our reason, and that this strange notion cannot be purged from our understanding of the temporal aspect of things without destroying our very understanding of those things, as being in time" (Taylor, 1963, p. 83) (3) $^{43}$

Taylor attempts to resolve the difficulties as follows: "What is needed", he writes, "... is some way of expressing statements embodying the idea of pure becoming, in such a way as to get rid of this idea altogether without thereby destroying the idea of time as well. This is actually attempted by substituting changeless dates for changing nows and thens, or substituting changeless temporal relations, such as earlier and later, for changing ones, such as past and future" (Taylor, 1963, p. 79). But as he points

\footnotetext{
42 A careful examination of the nature of our sense of temporal passage, including the circumstances or the way(s) in which we have it, should give us a much better conceptual understanding of the passage of time $_{2}$ than we now have.

43 My italics.
}

out on the same page, "whenever we reformulate any statement [that embodies the idea of temporal passage] so as to expurgate from it the idea of pure becoming, we are able to understand it only in the light of some other proposition that embodies the passage of time" (Taylor, 1963 , p. 78$)^{44}$. He considers four such propositions that embody the idea of "pure becoming" in them. Although I do not concur in every case with the reasons he gives why some or all of the reformulations he attempts are not equivalent to the original assertions - and if space permitted it would have been useful to consider these reasons ${ }^{45}$.

I agree that such attempts are bound to fail, if we understand by "pure becoming" what we ordinarily call the passage of time. For in light of our analysis of the everyday uses of 'time', 'time' and other temporal expression, it is seen that to "expurgate" the idea of temporal passage is to eliminate the idea of time 2 , from which the former idea is logically inseparable. Stated positively, it is impossible to eliminate the concept of the passage of time 2 by substituting either (a) "changeless dates for changing nows and thens", or (b) "changeless temporal relations, such as earlier and later, for changing ones, such as past and future".

Let us begin with (a). A date is changeless. The ideas of change and temporal passage do not apply to it. It is always what it is. For example, 2015 can never change into some other date, e.g.,

44 My italics.

45 To give just one example, he wrongly says that "....it ["now"] must ... be understood either as something that is changing, or else something in relation to which everything is changing" (Taylor, 1963, p. 81); though he is right in concluding that "it must, in short, be understood as a concept of pure becoming", i.e., a concept involving the concept of passage. What we should say instead is that it is either something that is passing - now $_{2}-$ or something in relation to which everything in the present is passing - now 
2016. Nor does it make sense to say that (the date $\left._{1}\right) 2015$ has come into existence on a certain day and will end on a certain other day. A date can and does pass. For instance, we say: "The date (month, year, decade, etc.) of my wife's and my $15^{\text {th }}$ wedding anniversary has passed". Indeed, the concept of a date presupposes the concept of the passage of time 2 Therefore only if one could reformulate statements involving the idea of temporal passage, without change or loss of meaning, into statements having reference to dates $_{1}$ but not (or not also) dates 2 , can the temporal passage be successfully expurgated. But that is impossible. The concept of temporal passage directly involves the concept of date (and, also, indirectly a date). As I pointed out, the concept of time $e_{2}$, hence the concept of temporal passage is presupposed by the concept of a date 2 .

Thus, though the concept of a date also presupposes the concept of a date, we cannot possibly translate statements involving dates 2 into equivalent statements involving dates ${ }_{1}$ but not temporal passage; any more than we can translate statements involving time in general into equivalent statements involving the concept of time 1 alone (without involving the concept of temporal passage).

Essentially the same logical state of affairs obtains with respect to the attempt to reformulate statements involving the concept of temporal passage into statements involving the concept of intervals of time (intervals) that widen with the passage of time; as opposed to fixed intervals (intervals $s_{1}$ ) that obtain between fixed dates $_{1}$ (e.g., as in "He travelled between 2014 and 2005"). We cannot reformulate, for example, " $X$ is receding ever farther into the past" as, say, " $X$ existed from July 1 through July 26, 2005" (Taylor, 1963, p. 81). The interval 1 between July 1 and July 26, 2005 is a fixed period that does not undergo change. It is composed of a certain number of weeks ${ }_{1}$, days ${ }_{1}$, etc., each of which is a fixed unit of time.

The time interval ${ }_{1}$ between a certain event, $A$, that took place on July 1, 2015 and an event $B$, that took place on July 25, 2015, does not itself pass; only July 1 and July 25, 2015 pass, recede into the past. (At the moment I am writing they have both receded into the past.) But even a time interval ${ }_{2}$ is not said to pass; though if it is an interval ${ }_{2}$ between some past event and the present (at time $t$ ), it constantly widens or increases, because the present $_{2}$ (at time $t$ ) itself passes into, become, the past ${ }^{46}$. But a fair amount of time ${ }_{2}$ did pass between the dates 2 separated by the interval ${ }_{1}$, i.e., here between July 1 and July 25 . Unless time 2 continued to pass after July 25, 2015 would never have come, and no interval between the two would have existed. (This is different from the false statement that, unless events continued to take place after July 1,2015 , that date would not possibly have come to pass.)

(b) Turning to (b), it can again be readily shown that 'earlier' and 'later' too cannot do the desired job. A sentence considered by Taylor, namely, "Y is receding ever farther into the past" (1), shows this. He rightly points out that this statement cannot be identified with "Y is earlier or anterior to some particular time [say 1960]" (Taylor, 1963, p. 82) ${ }^{47}[(2)]$. As he says, (2) is:

True of all times whatever, including those which are not receding into the past - namely, of all future times. We must identify the time to which $\mathrm{Y}$ is anterior, either as being the present time, or some time itself anterior to the present time, or some time itself anterior

\footnotetext{
46 Contrast Taylor, (Taylor, 1963, p. 81).

47 Italics in original.
} 
to the present, and the hopelessness of this is quite obvious. The time one would be thus calling "the present time" either has not always been present, but became such - in which case, though Y has indeed always been anterior to it, it is a time which undergoes the same pure becoming as $\mathrm{Y}^{48}$ - or that if in some sense "the present" time has always been the present time, and hence never became such ${ }^{49}$, then plainly $\mathrm{Y}$ has not always been anterior to it, but became such $^{50}$ (Taylor, 1963, p. 82).

But suppose we discover sentences involving the concept of temporal passage translatable without loss into sentences involving the concepts of earlier and later: would they show that the concept of temporal passage can be successfully expurgated? In light of our earlier analysis the answer is a definite "No". 'Earlier' and 'later' have a single sort of use, not corresponding specifically to 'time $e_{1}$, etc., or to 'time,' etc. They merely indicate a certain temporal sequence or order. Yet precisely because they indicate that, they logically presuppose the concept of time $e_{2}$ and the passage of time. For unless time passes nothing can exist or occur earlier or later than anything else (in time). For instance, these ideas cannot arise in relation to eternity; e.g., we cannot meaningfully speak (though theologians and philosophers are constantly tempted to speak) of one of God's states or actions as being before or after another state or action, etc. (Cf., mutatis mutandis, numbers.) Similarly with moments of time, hours, days, etc. Thus, even if e.g., "Y is receding ever farther into the past" were translatable into something like "Y is earlier than such and such a time (e.g., the present, or some

48 That is, our present 2.

49 That is, our present 2 .

50 Italics in original. past date)", the translation could not avoid the notion of temporal passage.

\section{REFERENCES}

Carroll, S. (2012). Mysteries of Modern Physics: Time. Chantilly, Virginia: The Teaching Company.

Hawking, S. W. (1994). Black Holes and Baby

Universes and other Essays. New York: Bantam Books.

Hawking, S. W. (2009). The Theory of Everything: The Origin and Fate of the Universe. Mumbai: JAICO Publishing House, Tenth Jaico Impression.

Khatchadourian, H. (1961, June). On Time. Philosophy and Phenomenological Research, 22(4), 456-466.

Khatchadourian, H. (1964, Fall). Relations. The Southern Journal of Philosophy, 2(3), 135-142.

Khatchadourian, H. (1967). A Critical Study in Method. The Hague: Martinus Nijhoff. Khatchadourian, H. (1973, September). Do Ordinary Spatial and Temporal Expressions Designate Relations? Philosophy and Phenomenological Research, 34 (1), 82-94.

Nagel, E. (1961). The Structure of Science: Problems in the Logic of Scientific Explanation. New York: Harcourt, Brace \& World.

Penrose, Roger (2012). Cycles of Time: An Extraordinary New View of the Universe. New York: Vintage Books, a Division of Random House, Inc.

Ryle, G. (1945). Philosophical Arguments. Oxford: Clarendon Press.

Taylor, R. (1963). Metaphysics. Englewood Cliffs, New Jersey: Prentice-Hall, Inc. 